\title{
A Method for Extracting Affine Invariant Local Features Using a Metric Tensor
}

\author{
[Takashi Toriu]
}

\begin{abstract}
This paper proposes a method for extracting local features which is invariant under affine transformations. This method is based on the metric tensor. Using the metric tensor, an affine invariant smoothing filter and a set of affine invariant differential operators are constructed. Then, combining them a set of affine invariant feature extractions is constructed. Effectiveness of the proposed method is confirmed by experiments. (Abstract)
\end{abstract}

Keywords-feature extracton, affine invariance, metric tensor, smoothing (key words)

\section{Introduction}

Affine invariance is important because images taken from different views are transformed with each other by affine transformation approximately. Therefore, it is desirable that features are invariant to the affine transformation. Affine transformation includes rotation, scaling, translation etc. As rotation invariant features, moments such as Zernike moment and Legendre moment are known [1-3]. In the paper [4], we proposed a method for extracting a set of rotaton invariant features based on 2 dimensional Herimite polynomials. A lot of methods have been propsed on affine invariant features[512]. Among them, the paper [5] describes affine invariant scale-space based on a nonlinear evolution equation. The paper [6] proposes a method to extend SIFT method [7] to be fully affine invariant.

In the previous paper [13], I proposed a new method for image smoothing that is invariant under affine transformations. Affine invariance is achieved by introducing a metric tensor with an appropriate transformational property against affine transformations. Affine invariance means that the result of smoothing after an affine transformation is the same as the result of the affine transformation after smoothing. The usual smoothing method by Gaussian filtering is not affine invariant.

In this paper, I propose a method for extracting local features which is invariant under affine transformations. This method is based on the metric tensor as the method of affine invariant smoothing mentioned above. We regard that feature extraction is a process of converting the input image to an image representing the local feature. Affine invariance means that the result of a feature extraction after an affine transformation is the same as the result of the affine transformation after the feature extraction. In other words, if affine transformation and feature extraction are commutable, the feature extraction is affine invariant.

Takashi Toriu

Centor for Research and Development of Higher Education, Osaka City University

Japan
The method proposed in this paper is based on the metric tensor. Using the metric tensor, first, an affine invariant smoothing filter is introduced. Next, a set of affine invariant differential operators are constructed. Then, combining them a set of affine invariant feature extractions is constructed. Effectiveness of the proposed method is confirmed by experiments.

\section{Affine Invariance}

\section{A. Definition}

We assume that the point $\left(x_{1}, x_{2}\right)$ is transformed to $\left(x_{1}^{\prime}, x_{2}^{\prime}\right)$ with an affine transformation

$$
\begin{aligned}
& x_{1}^{\prime}=a_{11} x_{1}+a_{12} x_{2}+b_{1}, \\
& x_{2}^{\prime}=a_{21} x_{1}+a_{22} x_{2}+b_{2} .
\end{aligned}
$$

We represent this transformation as $T$ and the above transformation (1) as

$$
\left(x_{1}, x_{2}\right) \stackrel{T}{\longrightarrow}\left(x_{1}^{\prime}, x_{2}^{\prime}\right)
$$

When the point $\left(x_{1}, x_{2}\right)$ on an image $I\left(x_{1}, x_{2}\right)$ is transformed to the point $\left(x_{1}^{\prime}, x_{2}^{\prime}\right)$ on another image $I_{T}\left(x_{1}, x_{2}\right)$ according to (1), and the following equation

$$
I\left(x_{1}, x_{2}\right)=I_{T}\left(x_{1}^{\prime}, x_{2}^{\prime}\right)
$$

is satisfied, we call $I_{T}\left(x_{1}, x_{2}\right)$ the affine transformation of $I\left(x_{1}, x_{2}\right)$. We represent it as

$$
I\left(x_{1}, x_{2}\right) \stackrel{T}{\longrightarrow} I_{T}\left(x_{1}, x_{2}\right)
$$

Let the image $I_{T}\left(x_{1}, x_{2}\right)$ be the affine transformation of an image $I\left(x_{1}, x_{2}\right)$ by $T$ as in (4). Let the $I_{F}\left(x_{1}, x_{2}\right)$ be the result of feature extraction of the image $I\left(x_{1}, x_{2}\right)$. We call $I_{F}\left(x_{1}, x_{2}\right)$ simply as the feature image of $I\left(x_{1}, x_{2}\right)$. Let $I_{T F}\left(x_{1}, x_{2}\right)$ be the feature image of $I_{T}\left(x_{1}, x_{2}\right)$, and $I_{F T}\left(x_{1}, x_{2}\right)$ be the result of the affine transformation of the feature image $I_{F}\left(x_{1}, x_{2}\right)$ as

$$
I_{F}\left(x_{1}, x_{2}\right) \stackrel{T}{\longrightarrow} I_{F T}\left(x_{1}, x_{2}\right)
$$


Based on these preparations, we define affine invariance of feature extraction as follows. If the following equation

$$
I_{F T}\left(x_{1}, x_{2}\right)=I_{T F}\left(x_{1}, x_{2}\right) \text {. }
$$

holds, we define this feature extraction as affine invariant feature extraction. According to this definition, if the feature extraction is affine invariant, the feature image after affine transformation and the affine transformation of the feature image are the same. In other words, affine invariance means that feature extraction and affine transformation are commutable.

\section{B. Metric Tensor}

In this subsection, we consider a method to construct a set of affine invariant feature images. For this purpose, we introduce an evaluation function $J\left(I\left(x_{1}, x_{2}\right), G\right)$ as

$$
J\left(I\left(x_{1}, x_{2}\right), G\right)=\iint \sum_{i, j} G_{i j}[I] \frac{\partial I}{\partial x_{i}} \frac{\partial I}{\partial x_{j}} d x_{1} d x_{2} / \sqrt{\operatorname{det}(G)},
$$

where $G$ is a metric tensor which satisfies following transformation property

$$
\begin{aligned}
& G_{i j}[I] \stackrel{T}{\longrightarrow} G_{i j}\left[I_{T}\right]=\sum_{k, l} a_{i k} a_{j l} G_{k l}[I], \\
& G\left[I_{T}\right]=A G[I] A^{t},
\end{aligned}
$$

where $A$ is the matrix element consist of $a_{i j} \mathrm{~s}$, and $A^{t}$ is the transpose of $A$. Here $\operatorname{det}(G)$ is the determinant of the matrix $G$, and $1 / \sqrt{\operatorname{det}(G[I])}$ is the area element. This evaluation function is affine invariant as is proved below.

\section{Theorem 1:}

Under the following affine transformation,

$$
\begin{aligned}
& I\left(x_{1}, x_{2}\right) \stackrel{T}{\longrightarrow} I_{T}\left(x_{1}, x_{2}\right), \\
& G_{i j}[I] \stackrel{T}{\longrightarrow} G_{i j}\left[I_{T}\right]=\sum_{k, l} a_{k i} a_{l j} G_{k l}[I],
\end{aligned}
$$

the evaluation function (7) is invariant as

$$
J\left(I\left(x_{1}, x_{2}\right), G\right)=J\left(I_{T}\left(x_{1}, x_{2}\right), G\left[I_{T}\right]\right),
$$

\section{Proof of the Theorem 1}

Differentiating both sides of (3) with respect to $x_{1}$ or $x_{2}$, we obtain

$$
\frac{\partial I\left(x_{1}, x_{2}\right)}{\partial x_{i}}=\sum_{k} \frac{\partial x_{k}^{\prime}}{\partial x_{i}} \frac{\partial I_{T}\left(x_{1}^{\prime}, x_{2}^{\prime}\right)}{\partial x_{k}^{\prime}}=\sum_{k} a_{k i} \frac{\partial I_{T}\left(x_{1}^{\prime}, x_{2}^{\prime}\right)}{\partial x_{k}^{\prime}}
$$

Using the relation on the area element under affine transformation,

$$
d x_{1}^{\prime} d x_{1}^{\prime}=\operatorname{det}\left(\begin{array}{ll}
\frac{\partial x_{1}^{\prime}}{\partial x_{1}} & \frac{\partial x_{1}^{\prime}}{\partial x_{2}} \\
\frac{\partial x_{2}^{\prime}}{\partial x_{1}} & \frac{\partial x_{2}^{\prime}}{\partial x_{1}}
\end{array}\right) d x_{1} d x_{2}
$$

we obtain

$$
\begin{aligned}
& J\left(I\left(x_{1}, x_{2}\right), G\right)=\iint \sum_{i, j} G_{i j}[I] \frac{\partial I}{\partial x_{i}} \frac{\partial I}{\partial x_{j}} d x_{1} d x_{2} / \sqrt{\operatorname{det}(G[I])} \\
& =\iint \sum_{i, j, k, l} G_{i j}[I] a_{k i} a_{l j} \frac{\partial I}{\partial x_{k}^{\prime}} \frac{\partial I}{\partial x_{l}^{\prime}} d x_{1} d x_{2} / \sqrt{\operatorname{det}(G[I])} \\
& =\iint \sum_{i, j} G_{i j}\left[I_{T}\right] \frac{\partial I}{\partial x_{i}^{\prime}} \frac{\partial I}{\partial x_{j}^{\prime}} d x_{1}^{\prime} d x_{2}^{\prime} / \operatorname{det}(A) \sqrt{\operatorname{det}\left(A^{-1} G\left[I_{T}\right] A^{t^{-1}}\right)} \\
& =\iint \sum_{i, j} G_{i j}\left[I_{T}\right] \frac{\partial I}{\partial x_{i}^{\prime}} \frac{\partial I}{\partial x_{j}^{\prime}} d x_{1}^{\prime} d x_{2}^{\prime} / \sqrt{\operatorname{det}\left(G\left[I_{T}\right]\right)} \\
& =J\left(I_{T}\left(x_{1}, x_{2}\right), G\left[I_{T}\right]\right),
\end{aligned}
$$

Thus, the theorem 1 is proved. From this theorem we can prove the following theorem.

\section{Theorem 2:}

The 2 by 2 symmetric matrix $G^{(M I N)}$ that minimizes the evaluation function (7) is transformed as follows under the affine transformation.

$$
\begin{aligned}
& I\left(x_{1}, x_{2}\right) \stackrel{T}{\longrightarrow} I_{T}\left(x_{1}, x_{2}\right), \\
& G^{(M I N)}{ }_{i j}[I] \stackrel{T}{\longrightarrow} G^{(M I N)}{ }_{i j}\left[I_{T}\right]=\sum_{k, l} a_{i k} a_{j l} G_{k l}^{(M I N)}[I] .
\end{aligned}
$$

This theorem means that if $G^{(M I N)}[I]$ minimizes the evaluation function $J\left(I\left(x_{1}, x_{2}\right), G\right), G^{(M I N)}\left[I_{T}\right]$ minimizes the evaluation function $J\left(I_{T}\left(x_{1}, x_{2}\right), G\right)$.

\section{Proof of theorem2:}

We assume that the minimum of $J\left(I\left(x_{1}, x_{2}\right), G\right)$ is

$$
\begin{aligned}
& J\left(I\left(x_{1}, x_{2}\right), G^{(M I N)}\right) \\
& =\iint \sum_{i, j} G_{i j}^{(M I N)}[I] \frac{\partial I}{\partial x_{i}} \frac{\partial I}{\partial x_{j}} d x_{1} d x_{2} / \sqrt{\operatorname{det}\left(G^{(M I N)}[I]\right)}=M I N
\end{aligned}
$$

Let $I_{T}\left(x_{1}, x_{2}\right)$ be the affine transformation of $I\left(x_{1}, x_{2}\right)$ and $G^{(M I N)}\left[I_{T}\right]$ be the affine transformation of $G^{(M I N)}[I]$. From the theorem 1, 


$$
J\left(I_{T}\left(x_{1}, x_{2}\right), G^{(M I N)}\left[I_{T}\right]\right)=J\left(I\left(x_{1}, x_{2}\right), G^{(M I N)}[I]\right)=M I N .
$$

This equation implies that $G^{(M I N)}\left[I_{T}\right]$ minimizes the evaluation function $J\left(I_{T}\left(x_{1}, x_{2}\right), G\right)$. In fact, if not, there exit $G_{\text {other }}$ such that $J\left(I_{T}\left(x_{1}, x_{2}\right), G_{\text {other }}\right)$ is less than MIN. Then, from the theorem $1, J\left(I\left(x_{1}, x_{2}\right), G_{o t h e r}\left[I_{T^{-1}}\right]\right)$ is also less than $M I N$ for $G_{o t h e r}\left[I_{T^{-1}}\right]$, that is inverse affine transformation of $G_{\text {other }}$. It is contrary to the assumption that the minimum value of $J\left(I\left(x_{1}, x_{2}\right), G\right)$ is $M I N$.

In this way, the following statement is proved: $G^{(M I N)}\left[I_{T}\right]$ minimizes $J\left(I_{T}\left(x_{1}, x_{2}\right), G_{T}\right)$. The metric tensor $G^{(M I N)}$ depends on the image $I\left(x_{1}, x_{2}\right)$. Therefore, we denote it as $G[I]$. When the image $I\left(x_{1}, x_{2}\right)$ is transformed to $I_{T}\left(x_{1}, x_{2}\right)$ by the affine transformation, $G[I]$ is transformed to $G\left[I_{T}\right]$ as in the equation (8). In the appendix A, we show how to obtain $G[I]$ which minimizes the evaluation function (7).

\section{c. Affine invariant differential operator}

Using the metric tensor $G[I]$, we define an affine invariant differential operator $H[I]$ as

$$
H[I]=\sum_{i, j} G_{i j}[I] \frac{\partial^{2}}{\partial x_{i} \partial x_{i}} .
$$

The differential operator $H[I]$ is a generalization of the usual Laplacian operator

$$
\nabla^{2}=\sum_{i} \frac{\partial^{2}}{\partial x_{i}^{2}}
$$

The image $I\left(x_{1}, x_{2}\right)$ is converted to

$$
I_{H[I]}\left(x_{1}, x_{2}\right)=H[I] I\left(x_{1}, x_{2}\right) .
$$

We can regard $I_{H[I]}\left(x_{1}, x_{2}\right)$ as an example of feature images.

\section{Theorem 3:}

The operator $H[I]$ is affine invariant. That is, the next relation holds.

$$
I_{H\left[I_{T}\right.}\left(x_{1}, x_{2}\right)=I_{T H\left[I_{T}\right]}\left(x_{1}, x_{2}\right) .
$$

\section{Proof of theorem 3:}

First, we notice that

$$
\begin{aligned}
& H[I]=\sum_{i, j} G_{i j}[I] \frac{\partial^{2}}{\partial x_{i} \partial x_{j}}=\sum_{i, j} G_{i j}[I] \frac{\partial x_{k}^{\prime}}{\partial x_{i}} \frac{\partial x_{l}^{\prime}}{\partial x_{j}} \frac{\partial^{2}}{\partial x_{k}^{\prime} \partial x_{l}^{\prime}} \\
& =\sum_{i, j} G_{i j}[I] a_{k i} a_{l j} \frac{\partial^{2}}{\partial x_{k}^{\prime} \partial x_{l}^{\prime}}
\end{aligned}
$$

Let $I_{T}\left(x_{1}, x_{2}\right)$ and $I_{H[I]_{T}}\left(x_{1}, x_{2}\right)$ be the affine transformations of $I\left(x_{1}, x_{2}\right)$ and $I_{H[I]}\left(x_{1}, x_{2}\right)$, respectively. Then, the relations

$$
I\left(x_{1}, x_{2}\right)=I_{T}\left(x_{1}^{\prime}, x_{2}^{\prime}\right), I_{H[I]}\left(x_{1}, x_{2}\right)=I_{H[I]_{T}}\left(x_{1}^{\prime}, x_{2}^{\prime}\right)
$$

hold for the affine transformation (1) (see (5)). From (17) and (19) we obtain

$$
\begin{aligned}
& I_{H[I]}\left(x_{1}, x_{2}\right)=H[I] I\left(x_{1}, x_{2}\right)=\sum_{i, j} G_{i j}[I] \frac{\partial^{2} I\left(x_{1}, x_{2}\right)}{\partial x_{i} \partial x_{j}} \\
& =\sum_{i, j, k, l} G_{i j}[I] a_{k i} a_{l j} \frac{\partial^{2} I_{T}\left(x_{1}^{\prime}, x_{2}^{\prime}\right)}{\partial x_{k}^{\prime} \partial x_{l}^{\prime}}=\sum_{k, l} G_{k l}\left[I_{T}\right] \frac{\partial^{2} I_{T}\left(x_{1}^{\prime}, x_{2}^{\prime}\right)}{\partial x_{k}^{\prime} \partial x_{l}^{\prime}} \\
& =H\left[I_{T}\right] I_{T}\left(x^{\prime}, y^{\prime}\right)=I_{T H\left[I_{T}\right]}\left(x^{\prime}, y^{\prime}\right),
\end{aligned}
$$

where $H\left[I_{T}\right]$ is

$$
H\left[I_{T}\right]=\sum_{k, l} G_{k l}\left[I_{T}\right] \frac{\partial^{2}}{\partial x_{k}^{\prime} \partial x_{l}^{\prime}}
$$

From (22) and (23), the relation (20) is confirmed. Thus, we see that the differential operator and the affine transformation is commutable. In other words, the differential operator can be regarded as an affine invariant feature extraction.

In order to construct a set of affine invariant feature extractions, we consider powers $H[I]^{n}$ of the differential operator $H[I]$.

\section{Theorem 4:}

The operator $H[I]^{n}$ is affine invariant. That is the next relation holds.

$$
I_{H[I]_{T}^{n}}\left(x_{1}, x_{2}\right)=I_{T H\left[I_{T}\right]^{n}}\left(x_{1}, x_{2}\right) .
$$

\section{Proof of theorem 4:}

The theorem 4 is proved by mathematical induction. When $n=1, H[I]^{n}$ is affine invariant from the theorem 3. We will 
prove below that $H[I]^{n+1}$ is affine invariant when $H[I]^{n}$ is affine invariant. First, we notice that

$$
I_{H[I]^{n}}\left(x_{1}, x_{2}\right)=I_{H[I]^{n}}\left(x_{1}^{\prime}, x_{2}^{\prime}\right) .
$$

Apply $H[I]$ to the left-hand side of (23), we get

$$
\begin{aligned}
& I_{H[I]^{n+1}}\left(x_{1}, x_{2}\right)=H[I] I_{H[I]^{n}}\left(x_{1}, x_{2}\right)=\sum_{i, j} G_{i j}[I] \frac{\partial^{2} I_{H[I]^{n}}\left(x_{1}, x_{2}\right)}{\partial x_{i} \partial x_{i}} \\
& =\sum_{i, j, k, l} G_{i j}[I] a_{k i} a_{l j} \frac{\partial^{2} I_{H[I]^{n}}\left(x_{1}^{\prime}, x_{2}^{\prime}\right)}{\partial x_{k}^{\prime} \partial x_{l}^{\prime}} \\
& =\sum_{k, l} G_{k l}\left[I_{T}\right] \frac{\partial^{2} I_{H[I]^{n} T}\left(x_{1}^{\prime}, x_{2}^{\prime}\right)}{\partial x_{k}^{\prime} \partial x_{l}^{\prime}} \\
& =H\left[I_{T} I_{H_{T}{ }^{n} T}\left(x^{\prime}, y^{\prime}\right)=H\left[I_{T}\right] I_{T H\left[I_{T}\right]^{n}}\left(x^{\prime}, y^{\prime}\right)=I_{T H\left[I_{T}\right]^{n+1}}\left(x^{\prime}, y^{\prime}\right) .\right.
\end{aligned}
$$

This equation implies (22). Thus the theorem is proved.

Next, we consider the following differential equation:

$$
\frac{d I\left(x_{1}, x_{2}, t\right)}{d t}=H\left[I\left(x_{1}, x_{2}, 0\right)\right] I\left(x_{1}, x_{2}, t\right) .
$$

Equation (25) is an anisotropic diffusion equation. If we set initial condition to be

$$
I\left(x_{1}, x_{2}, 0\right)=I\left(x_{1}, x_{2}\right),
$$

the solution $I\left(x_{1}, x_{2}, t\right)$ of (25) can be represented formally as

$$
I\left(x_{1}, x_{2}, t\right)=K[I, t] I\left(x_{1}, x_{2}\right),
$$

where $K[I, t]=\exp (H[I] t)$. The solution $I\left(x_{1}, x_{2}, t\right)$ is considered to be the result of applying the operator $K[I, t]$ to the image $I\left(x_{1}, x_{2}\right)$.

\section{Theorem 5:}

The operator $K[I, t]$ is affine invariant. That is, the next relation holds.

$$
I_{K[I, t]_{T}}\left(x_{1}, x_{2}\right)=I_{T K\left[I_{T}, t\right]}\left(x_{1}, x_{2}\right) .
$$

The proof of this theorem is seen in the appendix B.

The operator $K[I, t]$ can be regarded as a kind of smoothing operators. It may be valuable to recall the case of Gaussian smoothing. We consider the following diffusion equation.

$$
\frac{d I\left(x_{1}, x_{2}, t\right)}{d t}=\left(\frac{\partial^{2}}{\partial x_{1}^{2}}+\frac{\partial^{2}}{\partial x_{2}^{2}}\right) I .
$$

The solution is expressed as

$$
\begin{aligned}
& I\left(x_{1}, x_{2}, t\right) \\
& =\frac{1}{4 \pi t} \iint I\left(x_{1}^{\prime}, x_{2}^{\prime}, 0\right) \exp \left(-\frac{\left(x_{1}-x_{1}^{\prime}\right)^{2}+\left(x_{2}-x_{2}^{\prime}\right)^{2}}{4 t^{2}}\right) d x_{1}^{\prime} d x_{2}^{\prime} .
\end{aligned}
$$

From this analogy, we can regard the smoothing by equation (27) to be natural extension of Gaussian smoothing, but it is affine invariant.

\section{A Set of Affine Invariant features}

The differential operator $H[I]^{n}$ has property of a highpass filter and the smoothing operator $K[I, t]$ has property of a low-pass filter. Combining them, we can construct a set of affine invariant local features which have property of a bandpass filter. Band-pass property would be desirable for feature extraction. Since both of $H[I]^{n}$ and $K[I, t]$ are affine invariant, the combinations also are affine invariant. Thus, we obtain a set of affine invariant local features.

More specifically, the set of affine invariant local features are constructed as follows. First, the metric tensor $G[I]$ specific to the input image $I(x, y)$ is generated by the method described in the appendix B. Then, the image $I(x, y)$ is smoothed by solving the differential equation (25). The solution is represented formally as (27). Next, the smoothed image is successively processed by the differential operator $H[I]$ and the series of images

$H[I] K[I, t] I\left(x_{1}, x_{2}\right), H^{2}[I] K[I, t] I\left(x_{1}, x_{2}\right), \cdots H^{n}[I] K[I, t] I\left(x_{1}, x_{2}\right), \cdots$

are results of affine invariant feature extraction.

\section{Experiments and Results}

We conducted experiments to confirm effectiveness of our method. We used a following synthesized image of $512 \times 512$ pixels as an input image:

$$
I(x, y)=\exp \left(-\left(\frac{x^{2}}{2 \sigma_{x}^{2}}+\frac{y^{2}}{2 \sigma_{y}^{2}}\right)\right) .
$$

where $\sigma_{x}$ and $\sigma_{y}$ are randomly determined according to uniform distribution between 10.0 and 20.0. We prepared 64 images. These images were affine transformed, where affine transformation matrix $A$ s are determined randomly as 


$$
A=\left[\begin{array}{cc}
1+0.5\left(r_{11}-0.5\right) & 0.8\left(r_{12}-0.5\right) \\
0.8\left(r_{21}-0.5\right) & 1+0.5\left(r_{22}-0.5\right)
\end{array}\right],
$$

where $r_{11}, r_{12}, r_{21}$, and $r_{22}$ are random variables uniformly distributed between 0.0 and 1.0 .

Using these data, we conducted experiment to compare two cases. One is the conventional method where the metric tensor is constant and has values $g_{11}=g_{22}=1$ and $g_{12}=g_{21}=0$. In this case, the image is isotropically smoothed and differentiated by the usual Laplacian filter successively. The other is the proposed method

An example is shown in Figure 1 for the usual method. In the figure, the top-left represents the input image. The topright represents the affine transformed image. The feature images are calculated for these images. The feature image of the input image is shown at middle-left and the feature image after affine transformation is shown at middle-right of the figure 1 . The result of the affine transformation after feature extraction is shown at bottom right. The feature image after affine transformation and the affine transformed image after feature extraction is not the same in this case. It means that the feature extraction is not affine invariant.

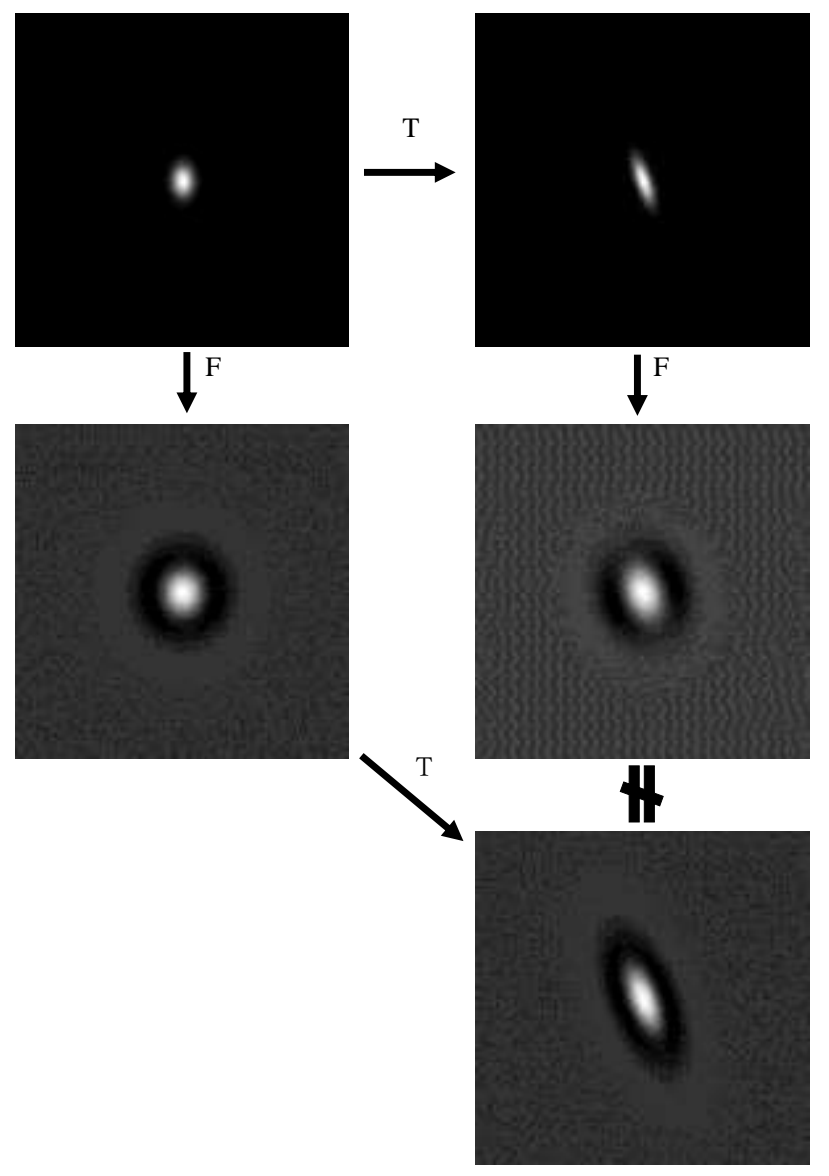

Figure 1. An example of the experiments in the case of the conventional method.
Another example is shown in Figure 2 for the usual method. In the figure, the top-left and the top-right represent the input image and the affine transformed image, respectively. The feature image of the input image is shown at middle-left and the feature image after affine transformation is shown at middle-right of the figure 2 . The result of the affine transformation after feature extraction is shown at bottom right. The feature image after affine transformation and the affine transformed image after feature extraction is the same in this case. It means that the feature extraction by the proposed method is affine invariant.

We evaluated average pixel error (mean square root error) for 64 times trials each between two images; one is the result of feature extraction after affine transformation and the other is the result of affine transformation after feature extraction. The result is shown in figure 3.The horizontal axis represents $\sigma=\sqrt{2} t$, where $t$ is the parameter in (27). From the figure, we can see that the error is significantly small in the case of the proposed method compared with the case of the conventional method. The error is normalized by the standard deviation of the pixel values of the feature image. From the theorem declared in this paper, the error should be exactly zero mathematically. The error observed in the experiment is considered to be from discretization error of finite sized images.

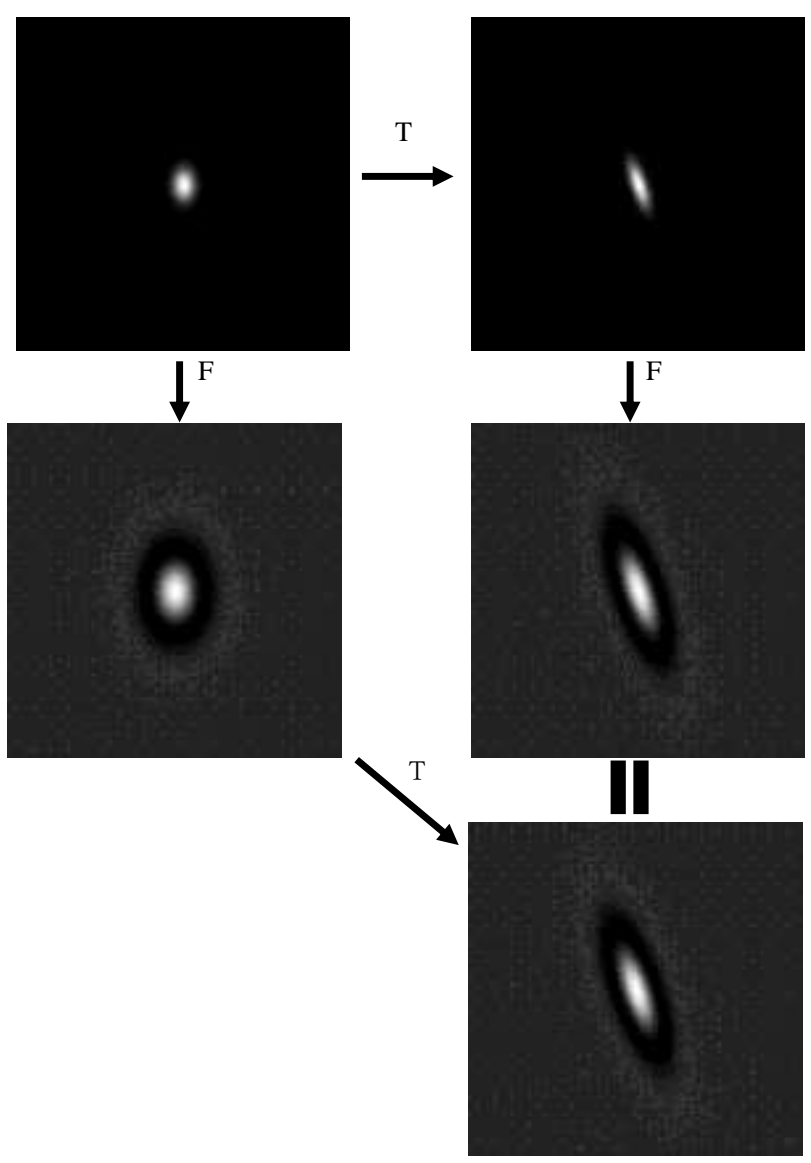

Figure 2. An example of the experiments in the case of the proposed of the method. 


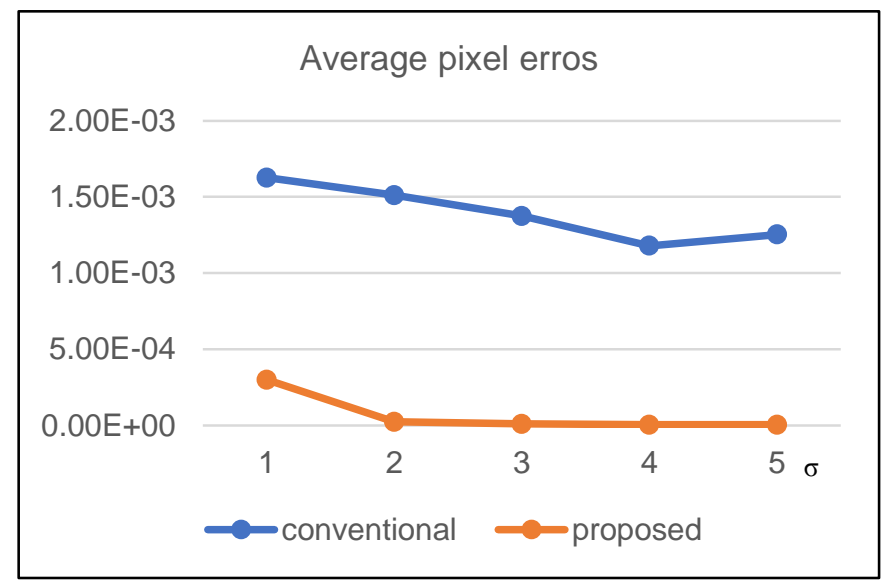

Figure 3. Average pixel error (mean square root error) between two images; one is the result of feature extraction after affine transformation and the other is the result of affine transformation after feature

\section{Conclusion}

In this paper, we proposed a method for extracting local features which is invariant under affine transformations using a metric tensor. We showed that an affine invariant smoothing filter and a set of affine invariant differential operators can be constructed using the metric tensor. Combining them, a set of affine invariant feature extractions was constructed. Feature extraction is affine invariant, if and only if affine transformation and feature extraction are commutable. We showed that this commutability is achieved in the proposed method by a computer simulation.

\section{Appendix A}

In this appendix, we show a method to obtain $G[I]$ that minimizes the following evaluation function.

$$
J\left(I\left(x_{1}, x_{2}\right), G\right)=\iint \sum_{i, j} G_{i j}[I] \frac{\partial I}{\partial x_{i}} \frac{\partial I}{\partial x_{j}} d x_{1} d x_{2} / \sqrt{\operatorname{det}(G)},
$$

Since equation (A-1) does not change by replacing $G[I]_{i j}$ with $k G[I]_{i j}$, we can only obtain the solution that minimize (A1) except scale factor. To fix the scale, we assume that determinant of $G$ is unit as

$$
G[I]_{11} G[I]_{22}-G[I]_{12}^{2}=1 .
$$

In this case, since the relation $G\left[I_{T}\right]=A G[I] A^{t}$ holds as is shown in (12), determinant of $A$ should be unit, because

$$
\operatorname{det} G\left[I_{T}\right]=\operatorname{det}\left(A G[I] A^{t}\right)=\operatorname{det} A \operatorname{det} G[I] \operatorname{det} A .
$$

Thus, affine transformation must be restricted to such transformation that does not change area of any region in the image. Then we again rewrite the evaluation function by introducing Lagrange multiplier as

$$
\begin{aligned}
& J\left(I\left(x_{1}, x_{2}\right), G\right) \\
& =\iint \sum_{i, j} G_{i j}[I] \frac{\partial I}{\partial x_{i}} \frac{\partial I}{\partial x_{j}} d x_{1} d x_{2} / \sqrt{\operatorname{det}(G)}-\lambda\left(G_{11}[I] G[I]_{22}-G_{12}[I]^{2}-1\right) .
\end{aligned}
$$

Differentiating (A-4) by $G_{11}[I], G_{22}[I]$ and $G_{12}[I]$, we obtain

$$
\begin{aligned}
& \frac{\partial J}{\partial G_{11}[I]}=\int\left(\frac{\partial I}{\partial x_{1}}\right)^{2} d x d y-\lambda G_{22}[I]=0, \\
& \frac{\partial J}{\partial G_{22}[I]}=\int\left(\frac{\partial I}{\partial x_{2}}\right)^{2} d x d y-\lambda G_{11}[I]=0, \\
& \frac{\partial J}{\partial G_{12}[I]}=2 \iint\left(\frac{\partial I}{\partial x_{1}}\right)\left(\frac{\partial I}{\partial x_{2}}\right) d x d y+2 \lambda G_{12}[I]=0 .
\end{aligned}
$$

Now we set $D_{11}, D_{22}$ and $D_{12}$ to be

$$
\begin{aligned}
& D_{11}=\iint\left(\frac{\partial I}{\partial x_{1}}\right)^{2} d x d y, \\
& D_{22}=\iint\left(\frac{\partial I}{\partial x_{2}}\right)^{2} d x d y, \\
& D_{12}=\iint\left(\frac{\partial I}{\partial x_{1}}\right)\left(\frac{\partial I}{\partial x_{2}}\right) d x d y,
\end{aligned}
$$

then we obtain

$$
\begin{aligned}
& G[I]_{22}=\iint\left(\frac{\partial I}{\partial x_{1}}\right)^{2} d x d y / \lambda=D_{11} / \lambda, \\
& G[I]_{11}=\iint\left(\frac{\partial I}{\partial x_{2}}\right)^{2} d x d y / \lambda=D_{22} / \lambda, \\
& G_{12}[I]=-\iint\left(\frac{\partial I}{\partial x_{1}}\right)\left(\frac{\partial I}{\partial x_{2}}\right) d x d y / \lambda=-D_{12} / \lambda .
\end{aligned}
$$

Since

$$
G_{11}[I] G_{22}[I]-G[I]_{12}{ }^{2}=\frac{D_{11} D_{22}-D_{12}^{2}}{\lambda^{2}}=1 .
$$

$\lambda$ is determined as

$$
\lambda=\sqrt{D_{11} D_{22}-D_{12}^{2}} .
$$

Therefore $G_{11}[I], G_{22}[I]$ and $G_{12}[I]$ are obtained as 


$$
\begin{aligned}
& G_{11}[I]=D_{22} / \sqrt{D_{11} D_{22}-D_{12}{ }^{2}}, \\
& G_{22}[I]=D_{11} / \sqrt{D_{11} D_{22}-D_{12}{ }^{2}}, \\
& G_{12}[I]=-D_{12} / \sqrt{D_{11} D_{22}-D_{12}{ }^{2}},
\end{aligned}
$$

Thus, we obtain $G_{11}[I], G_{22}[I]$ and $G_{12}[I]$ that minimize the evaluation function (A-1).

\section{Appendix B}

In this appendix, it is shown that the feature extraction $K[I, t]$ is affine invariant. First, we recall that if a feature extraction $F$ is affine invariant, next relation holds.

$$
I_{F T}\left(x_{1}, x_{2}\right)=I_{T F}\left(x_{1}, x_{2}\right)
$$

This is equivalent to

$$
I_{F}\left(x_{1}, x_{2}\right)=I_{T F T^{-1}}\left(x_{1}, x_{2}\right),
$$

where $T^{-1}$ is the inverse transformation of $T$. Therefore, feature extraction $F$ is affine invariant, if and only if the next relation holds.

$$
F=T F T^{-1},
$$

From this, we can prove the statement that if $H_{1}$ and $H_{2}$ are affine invariant, $H_{P}=H_{1}+H_{2}$ and $H_{M}=H_{1} H_{2}$ are also affine invariant. In fact, it is easy to see

$$
\begin{aligned}
& T H_{P} T^{-1}=T\left(H_{1}+H_{2}\right) T^{-1}=T H_{1} T^{-1}+T H_{2} T^{-1}=H_{1}+H_{2}=H_{P}, \\
& T H_{M} T^{-1}=T H_{1} H_{2} T^{-1}=T H_{1} T^{-1} T H_{2} T^{-1}=H_{1} H_{2}=H_{M} .
\end{aligned}
$$

In the same way, we obtain

$$
\begin{aligned}
& T K[I, t] T^{-1}=T \exp (H[I] t) T^{-1}=T \sum_{n} \frac{(H[I] t)^{n}}{n !} T^{-1} \\
& =\sum_{n} \frac{T(H[I] t)^{n} T^{-1}}{n !}=\sum_{n} \frac{\left(T H[I] T^{-1} t\right)^{n}}{n !}=\sum_{n} \frac{(H[I] t)^{n}}{n !}=K[I, t] .
\end{aligned}
$$

From this equation, we obtain

$$
T K[I, t]=K[I, t] T .
$$

This formula indicates that the feature extraction $K[I, t]$ is affine invariant.

\section{Acknowledgment}

This work was supported by JSPS KAKENHI Grant Number 17K00245.

\section{References}

[1] Y.-N. Hsu, H. H. Arsenault, and G. April Y. N. R. Nicole, "Rotationinvariant digital pattern recognition using circular harmonic expansion," Applied Optics, Vol. 21, No.22, 1982, pp. 4012-4015.

[2] C. - H. The and R. T. Chin, "On Image Analysis by the RotationInvariant Correlation Filters," IEEE Transaction, PAMI, Vol. 16, No. 2, 1994, pp. 496-513.

[3] G. Ravichandran; D. Casasent, "Advanced in-plane rotation-invariant correlation filters,", IEEE Transaction, PAMI, Vol. 16, No. 4, 1994, pp.415-420.

[4] T. Toriu, H. Hama, M. Miyazaki, K. Miyazaki and K. Toda, A Similar Trademark Retrieval System based on Rotation Invariant Local Features, Proc. of ICFSP, 2016.

[5] P. J. Olver, G. Sapirio and A. Tannenbaum, Affine Invariant Detection: Edge Maps, Anisotropic Diffusion, and Active Contours, Acta Applicandae Mathematicae 59, pp.45-77, 1999.

[6] J.-M. Morel and G. Yu, ASIFT: A New Framework for Fully Affine Invariant Image Comparison, SIAM J. Imaging Sci., 2(2), pp.438-469, 2009

[7] D.G.Lowe, Distinctive image features from scale-invariant keypoints, International Journal of Computer Vision,Vol.60, No.2, 91-110, 2004

[8] X. Qu, F. Zhao, M. Zhou, and H. Huo, A Novel Fast and Robust Binary Affine Invariant Descriptor for Image Matching, Mathematical Problems in Engineering, Vol. 2014, Article ID 129230, 7 pages.

[9] S.Leutenegger, M.Chli and R.Y.Siegwart BRISK: Binary Robust Invariant Scalable Keypoints, In Proc. International Conference on Computer Vision, 2011.

[10] N. Dalal, B. Triggs, Histograms of oriented gradients for human detection. In Proc. IEEE Conference on Computer Vision and Pattern Recognition, 886-893, 2005.

[11] K. Mikolajczyk and Cordelia Schmid, Scale \& Affine Invariant Interest Point Detector, Proc. of ECCV, pp.128-142, 2002.

[12] K. Mikolajczyk and Cordelia Schmid, An Affine Invariant Interest Point Detector, International Journal of Computer Vision 60(1), pp. 63-86, 2004.

[13] T. Toriu, T. T. Zin and H. Hama, Image Smoothing Using a Metric Tensor for an Affine Invariant Scale Space, 4th International Conference on Image Processing Theory, Tools and Applications, 2014.

About Author (s):

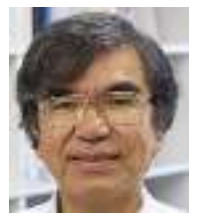

Takashi Toriu received the Dr. Sci. degrees in Physics from Kyoto University, Japan. $\mathrm{He}$ is currently specially appointed professor at Osaka City University. His research interests include image processing, computer vision, mechanism of visual attention. He is a member of IEEE, IEICE, ITE and IEEJ. 